\title{
Bimetallic Complexes of Metallacyclopentynes: Cis Vs Trans and Planarity Vs Non-planarity
}

\section{Marc A. Bach, ${ }^{a}$ Pattiyil Parameswaran, ${ }^{b}$ Eluvathingal D. Jemmis ${ }^{b}$ * and Uwe Rosenthal ${ }^{a}$}

\author{
Leibniz-Institut für Katalyse e.V. an der Universität Rostock, Albert-Einstein-Str. 29a \\ Rostock, Germany. Fax: +49-381-1281-5000; Tel: +49-381-1281-176 \\ E-mail: uwe.rosenthal@catalysis.de \\ Department of Inorganic and Physical Chemistry, Indian Institute of Science, Bangalore - \\ 560012, India, E-mail: jemmis@ipc.iisc.ernet.in
}

Total energy and Cartesian coordinates of all molecules obtained from of B3LYP/LANL2DZ level of theory using Gaussian 03 program package. NIF is the number of imaginary frequencies.

\section{$1 T i$}

Total Energy including ZPVE $=-599.621920 \mathrm{au}$ $\mathrm{NIF}=0$

$\begin{array}{lrcc}6 & 0.029788 & 1.585464 & 1.873267 \\ 1 & 0.962895 & 1.577310 & 2.433780 \\ 1 & -0.856870 & 1.628757 & 2.501394 \\ 6 & 0.010291 & 2.247571 & 0.633091 \\ 6 & -0.010392 & 2.247596 & -0.632604 \\ 6 & -0.029846 & 1.586149 & -1.873057 \\ 1 & -0.962996 & 1.577937 & -2.433485 \\ 1 & 0.856805 & 1.629653 & -2.501175 \\ 6 & 2.430937 & 0.291533 & 0.306462 \\ 1 & 2.862948 & 1.246556 & 0.571171 \\ 6 & 2.260145 & -0.208195 & -1.019167 \\ 1 & 2.554224 & 0.291952 & -1.929837 \\ 6 & 1.636248 & -1.490186 & -0.934200 \\ 1 & 1.376543 & -2.123727 & -1.771014 \\ 6 & 1.464980 & -1.803625 & 0.454614 \\ 1 & 1.046431 & -2.717037 & 0.851960 \\ 6 & 1.940239 & -0.701213 & 1.224787 \\ 1 & 1.967232 & -0.646294 & 2.303069 \\ 6 & -2.260337 & -0.208275 & 1.018946 \\ 1 & -2.554572 & 0.291823 & 1.929595 \\ 6 & -1.636343 & -1.490235 & 0.934075 \\ 1 & -1.376787 & -2.123796 & 1.770920 \\ 6 & -1.464843 & -1.803653 & -0.454712 \\ 1 & -1.046234 & -2.717052 & -0.852023 \\ 6 & -1.940003 & -0.701239 & -1.224954 \\ 1 & -1.966796 & -0.646269 & -2.303238 \\ 6 & -2.430911 & 0.291463 & -0.306692 \\ 1 & -2.862900 & 1.246473 & -0.571481 \\ 22 & 0.000016 & 0.110217 & 0.000056\end{array}$

\begin{tabular}{|c|c|c|c|}
\hline & $\begin{array}{l}\text { Energy in } \\
=0 \\
=0\end{array}$ & $\begin{array}{l}\text { luding } \\
6460 \text { au }\end{array}$ & \\
\hline 6 & -0.000039 & 1.677974 & -1.884176 \\
\hline 1 & -0.907448 & 1.744323 & -2.485627 \\
\hline 1 & 0.907255 & 1.744451 & -2.485781 \\
\hline 6 & -0.000086 & 2.365479 & -0.633043 \\
\hline 6 & 0.000334 & 2.365340 & 0.632812 \\
\hline 6 & 0.000210 & 1.678394 & 1.88 \\
\hline 1 & 0.907553 & 1.744627 & \\
\hline 1 & -0.907212 & 1.744888 & 85518 \\
\hline 6 & -2.574544 & 0.265186 & 060 \\
\hline 1 & -3.026771 & 1.248030 & -0.0 \\
\hline 6 & -2.238009 & -0.507104 & \\
\hline 1 & -2.402443 & -0.219316 & 2.1 \\
\hline 6 & -1.675747 & -1.745536 & 0.71 \\
\hline 1 & -1.344373 & -2.554780 & \\
\hline 6 & -1.675492 & -1.746329 & -0.7 \\
\hline 1 & -1.343865 & -2.556271 & -1.3 \\
\hline 6 & -2.237557 & -0.508373 & -1.1 \\
\hline 1 & -2.401614 & -0.221663 & -2.1 \\
\hline 6 & 2.237940 & -0.507571 & -1.16 \\
\hline 1 & 2.402442 & -0.220148 & -2.1899 \\
\hline 6 & 1.675662 & -1.745872 & -0.7 \\
\hline 1 & 1.344363 & -2.555342 & -1.35493 \\
\hline 6 & 1.675342 & -1.746208 & 0.71777 \\
\hline 1 & 1.343676 & -2.555952 & 1.35386 \\
\hline 6 & 2.237444 & -0.508140 & 1.16170 \\
\hline 1 & 2.401487 & -0.221158 & 2.19050 \\
\hline 6 & 2.574495 & 0.265056 & 0.00040 \\
\hline 1 & 3.026749 & 1.247886 & 0.00078 \\
\hline 40 & 0.000012 & 0.100416 & $-0.0000^{7}$ \\
\hline
\end{tabular}




\section{$2 \mathrm{Ti}$}

Total Energy including

ZPVE=-598.39480 a.u.

$\begin{array}{lll}22 & 0.0 .139627 & 0.000000\end{array}$

$\begin{array}{llll}6 & -0.007977 & 1.551453 & 1.740345\end{array}$

$\begin{array}{llll}1 & -0.013326 & 1.681539 & 2.813181\end{array}$

$\begin{array}{llll}6 & -0.002797 & 2.296001 & 0.672534\end{array}$

$\begin{array}{llll}6 & 0.003019 & 2.296396 & -0.671831\end{array}$

$\begin{array}{llll}6 & 0.007513 & 1.552534 & -1.740069\end{array}$

$\begin{array}{llll}1 & 0.010649 & 1.682939 & -2.812866\end{array}$

$\begin{array}{llll}6 & -2.083309 & -0.445262 & -1.178402\end{array}$

$\begin{array}{llll}6 & -1.540927 & -1.668693 & -0.690826\end{array}$

$\begin{array}{llll}6 & -1.565958 & -1.627316 & 0.747047\end{array}$

$\begin{array}{llll}6 & -2.128759 & -0.380095 & 1.142809\end{array}$

$\begin{array}{llll}6 & -2.426472 & 0.364382 & -0.044883\end{array}$

$\begin{array}{llll}1 & -2.210554 & -0.173795 & -2.216347\end{array}$

$\begin{array}{llll}1 & -1.197577 & -2.495247 & -1.296108\end{array}$

$\begin{array}{llll}1 & -1.245513 & -2.416279 & 1.412094\end{array}$

$\begin{array}{llll}1 & -2.291945 & -0.049133 & 2.158200\end{array}$

$\begin{array}{llll}1 & -2.839274 & 1.362662 & -0.080535\end{array}$

$\begin{array}{llll}6 & 2.127241 & -0.382056 & -1.144070\end{array}$

$\begin{array}{rrrr}6 & 2.425926 & 0.365148 & 0.041753\end{array}$

$\begin{array}{llll}6 & 2.084737 & -0.442344 & 1.177364\end{array}$

$\begin{array}{llll}6 & 1.542282 & -1.667059 & 0.693083\end{array}$

$\begin{array}{llll}6 & 1.565907 & -1.628785 & -0.744890\end{array}$

$\begin{array}{llll}1 & 2.288944 & -0.053446 & -2.160458\end{array}$

$\begin{array}{llll}1 & 2.838096 & 1.363768 & 0.075077\end{array}$

$\begin{array}{llll}1 & 2.212896 & -0.168540 & 2.214575\end{array}$

$\begin{array}{llll}1 & 1.199980 & -2.492606 & 1.300355\end{array}$

$\begin{array}{lllll}1 & 1.245429 & -2.419484 & -1.407852\end{array}$

\section{Zr}

Total Energy including

ZPVE=-586.93085 a.u.

$\begin{array}{llll}6 & -1.767476 & 1.685271 & 0.000000\end{array}$

$\begin{array}{llll}40 & 0.00 & 0.131582 & 0.000000\end{array}$

$\begin{array}{rrrr}6 & -1.159447 & -0.483717 & 2.246412 \\ 6 & 1.771144 & 1.681707 & 0.000000 \\ 6 & 1.164002 & -0.500792 & 2.238619 \\ 6 & 0.008939 & 0.279630 & 2.578565 \\ 1 & -1.370901 & -2.521121 & 1.334835 \\ 1 & 1.339171 & -2.541610 & 1.327224 \\ 1 & -1.370901 & -2.521121 & -1.334835 \\ 6 & 1.164002 & -0.500792 & -2.238619 \\ 6 & 0.710934 & -1.729129 & -1.666701 \\ 1 & 0.017637 & 1.265734 & -3.023450 \\ 1 & -2.184402 & -0.177533 & -2.402601 \\ 1 & 1.339171 & -2.541610 & -1.327224 \\ 6 & -0.728453 & -1.718379 & -1.670954 \\ 6 & -1.159447 & -0.483717 & -2.246412\end{array}$

$\begin{array}{llll}1 & 2.194308 & -0.209965 & -2.388913\end{array}$

$\begin{array}{llll}6 & 0.008939 & 0.279630 & -2.578565\end{array}$

$\begin{array}{llll}1 & 2.194308 & -0.209965 & 2.388913\end{array}$

$\begin{array}{llll}1 & 0.017637 & 1.265734 & 3.023450\end{array}$

$\begin{array}{llll}6 & -0.728453 & -1.718379 & 1.670954\end{array}$

$\begin{array}{llll}6 & 0.710934 & -1.729129 & 1.666701\end{array}$

$\begin{array}{llll}1 & -2.184402 & -0.177533 & 2.402601\end{array}$

$\begin{array}{llll}1 & 2.829588 & 1.914369 & 0.000000\end{array}$

$\begin{array}{llll}6 & -0.668299 & 2.408931 & 0.000000\end{array}$

$\begin{array}{llll}1 & -2.825388 & 1.920430 & 0.000000\end{array}$

$\begin{array}{llll}6 & 0.673380 & 2.407350 & 0.000000\end{array}$

\section{Ti-Ni}

Total Energy including ZPVE = -785.462941 au $\mathrm{NIF}=0$

$\begin{array}{lccc}6 & 0.315734 & -1.565045 & -1.081647 \\ 1 & 0.063032 & -2.505022 & -0.586731 \\ 1 & 0.645605 & -1.725872 & -2.107182 \\ 6 & -0.594993 & -0.463519 & -0.861593 \\ 6 & -0.599538 & 0.826285 & -0.518523 \\ 6 & 0.314602 & 1.888747 & -0.168559 \\ 1 & 0.677581 & 2.503207 & -0.992018 \\ 1 & 0.053486 & 2.496576 & 0.699563 \\ 6 & 0.452381 & -1.104607 & 1.965517 \\ 1 & -0.517829 & -1.558169 & 1.824735 \\ 6 & 0.684225 & 0.249076 & 2.347299 \\ 1 & -0.078161 & 0.982386 & 2.565076 \\ 6 & 2.096862 & 0.475378 & 2.349952 \\ 1 & 2.592290 & 1.405348 & 2.594284 \\ 6 & 2.736372 & -0.757770 & 2.000552 \\ 1 & 3.801505 & -0.923980 & 1.941025 \\ 6 & 1.721051 & -1.729849 & 1.733684 \\ 1 & 1.882461 & -2.755436 & 1.433048 \\ 6 & 2.932294 & -0.606320 & -1.988699 \\ 1 & 2.710151 & -1.470794 & -2.595781 \\ 6 & 3.805152 & -0.590292 & -0.866154 \\ 1 & 4.346260 & -1.440743 & -0.474683 \\ 6 & 3.873570 & 0.760786 & -0.378098 \\ 1 & 4.467452 & 1.108062 & 0.456096 \\ 6 & 3.029123 & 1.566586 & -1.187495 \\ 1 & 2.886671 & 2.631656 & -1.080795 \\ 6 & 2.412241 & 0.715684 & -2.169768 \\ 1 & 1.718602 & 1.028378 & -2.938866 \\ 1 & -2.971619 & 2.835851 & 1.235748 \\ 1 & -4.055020 & 2.710193 & -0.613554 \\ 1 & -4.840427 & 1.779502 & 1.154002 \\ 1 & -4.700034 & -2.182096 & 0.380712 \\ 1 & -4.367840 & -1.927069 & -1.725174 \\ 1 & -2.978346 & -3.068146 & -0.551080 \\ 15 & -3.596525 & 1.824523 & 0.424635\end{array}$




$\begin{array}{rrrr}15 & -3.627458 & -1.784885 & -0.498014 \\ 22 & 1.527679 & -0.007323 & 0.000675 \\ 28 & -2.344981 & 0.058995 & -0.235407\end{array}$

\section{Zr-Ni}

Total Energy including ZPVE = -773.999924 au $\mathrm{NIF}=0$

$\begin{array}{lrrc}6 & 0.103527 & -0.748658 & 1.823024 \\ 1 & -0.235506 & -0.121593 & 2.650917 \\ 1 & 0.487769 & -1.705306 & 2.186951 \\ 6 & -0.789019 & -0.812556 & 0.670222 \\ 6 & -0.789064 & -0.813707 & -0.668924 \\ 6 & 0.103414 & -0.751548 & -1.821881 \\ 1 & 0.487609 & -1.708730 & -2.184451 \\ 1 & -0.235712 & -0.125712 & -2.650673 \\ 6 & 0.157299 & 2.296881 & -0.002698 \\ 1 & -0.923027 & 2.270025 & -0.003474 \\ 6 & 0.998080 & 2.303636 & -1.163376 \\ 1 & 0.665960 & 2.319456 & -2.191839 \\ 6 & 2.359614 & 2.314321 & -0.718119 \\ 1 & 3.234415 & 2.348213 & -1.353442 \\ 6 & 2.358532 & 2.315420 & 0.716014 \\ 1 & 3.232363 & 2.350265 & 1.352624 \\ 6 & 0.996334 & 2.305448 & 1.159221 \\ 1 & 0.662645 & 2.322818 & 2.187148 \\ 6 & 2.996874 & -1.709061 & 1.162130 \\ 1 & 2.813035 & -1.981143 & 2.191652 \\ 6 & 3.838297 & -0.645251 & 0.719415 \\ 1 & 4.408318 & 0.019413 & 1.354779 \\ 6 & 3.838083 & -0.646772 & -0.71894 \\ 1 & 4.407960 & 0.016501 & -1.35588 \\ 6 & 2.996567 & -1.711553 & -1.15914 \\ 1 & 2.812454 & -1.985838 & -2.18803 \\ 6 & 2.452174 & -2.356061 & 0.002251 \\ 1 & 1.780169 & -3.204821 & 0.003252 \\ 1 & -3.121034 & 0.212958 & -3.101409 \\ 1 & -4.584560 & -1.146490 & -2.312048 \\ 1 & -4.818568 & 0.967427 & -2.020087 \\ 1 & -4.819836 & 0.969868 & 2.017741 \\ 1 & -4.582925 & -1.142982 & 2.315142 \\ 1 & -3.121092 & 0.220497 & 3.100682 \\ 15 & -3.792946 & -0.033654 & -1.852850 \\ 15 & -3.792914 & -0.030275 & 1.852905 \\ 40 & 1.403976 & 0.015503 & -0.000075 \\ 28 & -2.523066 & -0.307116 & 0.000268\end{array}$

Total Energy including ZPVE $=-1044.591134$ $\mathrm{NIF}=0$

$\begin{array}{lrrc}6 & 0.280317 & -0.164183 & -0.668408 \\ 6 & 0.281385 & -0.152355 & 0.675898 \\ 6 & -0.731193 & -0.206563 & -1.736221 \\ 6 & -0.731469 & -0.171397 & 1.742468 \\ 22 & -2.066973 & -0.012798 & -0.000762 \\ 22 & 2.232389 & -0.028987 & 0.005287 \\ 1 & -0.675797 & 0.628461 & -2.441478 \\ 1 & -0.748665 & -1.145572 & -2.294444 \\ 1 & -0.795499 & -1.128064 & 2.268426 \\ 1 & -0.642998 & 0.634841 & 2.475489 \\ 6 & -2.185407 & -2.460364 & 0.281926 \\ 1 & -1.281829 & -3.016911 & 0.489983 \\ 6 & -3.065218 & -1.885601 & 1.262031 \\ 1 & -2.946647 & -1.932188 & 2.334565 \\ 6 & -4.129404 & -1.245821 & 0.569971 \\ 1 & -4.957124 & -0.723075 & 1.027860 \\ 6 & -2.736831 & -2.203225 & -1.010856 \\ 1 & -2.336248 & -2.540461 & -1.954486 \\ 6 & -3.916312 & -1.421288 & -0.841117 \\ 1 & -4.559565 & -1.067971 & -1.635020 \\ 6 & -1.678455 & 2.393133 & -0.474124 \\ 1 & -0.717078 & 2.713562 & -0.845996 \\ 6 & -2.789472 & 1.966743 & -1.274645 \\ 1 & -2.819084 & 1.920812 & -2.353850 \\ 6 & -3.858259 & 1.636668 & -0.390970 \\ 1 & -4.840819 & 1.298206 & -0.685925 \\ 6 & -2.063748 & 2.320426 & 0.897177 \\ 1 & -1.454184 & 2.591468 & 1.746271 \\ 6 & -3.402436 & 1.823942 & 0.955516 \\ 1 & -3.978256 & 1.660608 & 1.856018 \\ 6 & 2.081890 & -2.570150 & -0.210036 \\ 1 & 1.123362 & -3.039152 & -0.381492 \\ 6 & 2.960026 & -2.085798 & -1.224318 \\ 1 & 2.811176 & -2.180081 & -2.292620 \\ 6 & 4.087057 & -1.473409 & -0.578390 \\ 1 & 4.948106 & -1.047918 & -1.074360 \\ 6 & 2.623623 & -2.220760 & 1.062681 \\ 1 & 2.186788 & -2.447704 & 2.025956 \\ 6 & 3.877435 & -1.547720 & 0.836519 \\ 1 & 4.563144 & -1.209727 & 1.601563 \\ 6 & 1.939515 & 2.341531 & 0.549169 \\ 1 & 1.023195 & 2.662058 & 1.021823 \\ 6 & 3.133715 & 1.941259 & 1.227762 \\ 1 & 3.275787 & 1.915109 & 2.301066 \\ 6 & 4.112714 & 1.600896 & 0.241848 \\ 1 & 5.117922 & 1.254460 & 0.438770 \\ 6 & 2.165205 & 2.216072 & -0.860279 \\ 1 & 1.468935 & 2.465053 & -1.648262\end{array}$




$\begin{array}{llll}6 & 3.511869 & 1.748059 & -1.047065 \\ 1 & 3.996948 & 1.572883 & -1.998608\end{array}$

\section{Zr-Zr}

Total Energy including ZPVE $=-1021.636795 \mathrm{au}$ $\mathrm{NIF}=0$$$
6
$$

- $253994-0.360965$

$\begin{array}{llll}6 & -0.253474 & -0.351427 & -0.8\end{array}$

$\begin{array}{llll}6 & 0.776214 & -0.501495 & 1.752576\end{array}$

$\begin{array}{lllll}6 & 0.776297 & -0.472298 & -1.761756\end{array}$

$\begin{array}{lllll}40 & 2.198294 & -0.033839 & 0.001129\end{array}$

$\begin{array}{lllll}40 & -2.326673 & -0.061967 & -0.006830\end{array}$

$\begin{array}{llll}1 & 0.610996 & 0.150809 & 2.616612\end{array}$

$\begin{array}{llll}1 & 0.895479 & -1.533981 & 2.103152\end{array}$

$\begin{array}{llll}1 & 0.925649 & -1.504127 & -2.103949\end{array}$

$\begin{array}{lllll}1 & 0.597301 & 0.168728 & -2.630606\end{array}$

$\begin{array}{lllll}6 & 2.777838 & -2.577431 & -0.120684\end{array}$

$\begin{array}{lllll}1 & 1.967456 & -3.290185 & -0.208086\end{array}$

$\begin{array}{lllll}6 & 3.481860 & -1.964693 & -1.211699\end{array}$

$\begin{array}{lllll}1 & 3.297901 & -2.130963 & -2.263975\end{array}$

$\begin{array}{lllll}6 & 4.472910 & -1.097703 & -0.663092\end{array}$

$\begin{array}{lllll}1 & 5.179734 & -0.503383 & -1.226862\end{array}$

$\begin{array}{lllll}6 & 3.364201 & -2.114511 & 1.102038\end{array}$

$\begin{array}{llll}1 & 3.080844 & -2.414421 & 2.100767\end{array}$

$\begin{array}{lllll}6 & 4.396668 & -1.185983 & 0.772206\end{array}$

$\begin{array}{lllll}1 & 5.036541 & -0.674259 & 1.478633\end{array}$

$\begin{array}{llll}6 & 1.532090 & 2.446916 & 0.471902\end{array}$

$\begin{array}{lllll}1 & 0.547886 & 2.640467 & 0.869023\end{array}$

$\begin{array}{llll}6 & 2.707019 & 2.188290 & 1.248951\end{array}$

$\begin{array}{llll}1 & 2.764224 & 2.172139 & 2.328983\end{array}$

$\begin{array}{llll}6 & 3.794202 & 1.981240 & 0.340445\end{array}$

$\begin{array}{llll}1 & 4.822499 & 1.788078 & 0.613078\end{array}$

$\begin{array}{llll}6 & 1.889353 & 2.395733 & -0.912960\end{array}$

$\begin{array}{lllll}1 & 1.228614 & 2.554317 & -1.753582\end{array}$

$\begin{array}{lllll}6 & 3.286550 & 2.096004 & -0.996193\end{array}$

$\begin{array}{lllll}1 & 3.861835 & 2.003405 & -1.908181\end{array}$

$\begin{array}{llll}6 & -2.406113 & -2.704910 & 0.231173\end{array}$

$\begin{array}{lllll}1 & -1.480714 & -3.227334 & 0.430143\end{array}$

$\begin{array}{lllll}6 & -3.295988 & -2.179180 & 1.218118\end{array}$

$\begin{array}{lllll}1 & -3.186157 & -2.281954 & 2.290877\end{array}$

$\begin{array}{lllll}6 & -4.386505 & -1.535912 & 0.536125\end{array}$

$\begin{array}{llll}1 & -5.247018 & -1.076400 & 1.004554\end{array}$

$\begin{array}{llll}6 & -2.913511 & -2.363962 & -1.060816\end{array}$

$\begin{array}{lllll}1 & -2.470880 & -2.636599 & -2.010009\end{array}$

$\begin{array}{lllll}6 & -4.148055 & -1.641743 & -0.872703\end{array}$

$\begin{array}{lllll}1 & -4.808796 & -1.294579 & -1.657116\end{array}$

$\begin{array}{llll}6 & -1.969553 & 2.466253 & -0.507689\end{array}$

$\begin{array}{lllll}1 & -1.038741 & 2.779259 & -0.956597\end{array}$

$\begin{array}{lllll}6 & -3.163446 & 2.113779 & -1.216571\end{array}$

$\begin{array}{lrrr}1 & -3.289370 & 2.125299 & -2.292742 \\ 6 & -4.177432 & 1.795862 & -0.255243 \\ 1 & -5.196505 & 1.508044 & -0.477491 \\ 6 & -2.233810 & 2.342216 & 0.896476 \\ 1 & -1.553416 & 2.579165 & 1.702885 \\ 6 & -3.601564 & 1.918638 & 1.049532 \\ 1 & -4.120778 & 1.774419 & 1.989024\end{array}$

\section{Ti-Ni}

Total Energy including $\mathrm{ZPVE}=-785.439200 \mathrm{au}$ $\mathrm{NIF}=0$

$\begin{array}{lccc}6 & 1.201747 & -1.380903 & -1.390641 \\ 1 & 1.223129 & -1.118422 & -2.452745 \\ 1 & 1.552109 & -2.398916 & -1.190268 \\ 28 & 2.240824 & -0.373489 & 0.006580 \\ 6 & 0.443259 & -0.710950 & 0.759093 \\ 6 & 0.081470 & -0.911434 & -0.576206 \\ 22 & -1.593166 & 0.054260 & 0.048768 \\ 6 & -0.417920 & -0.139481 & 1.839634 \\ 1 & -0.732055 & -0.851598 & 2.607615 \\ 1 & -0.041792 & 0.778462 & 2.301814 \\ 6 & -0.934355 & 1.731059 & -1.633436 \\ 6 & -2.357998 & 1.805064 & -1.540185 \\ 6 & -2.698196 & 2.268364 & -0.229076 \\ 6 & -1.485218 & 2.483637 & 0.497044 \\ 6 & -0.397528 & 2.155826 & -0.372622 \\ 1 & -0.367225 & 1.426334 & -2.501482 \\ 1 & -3.057208 & 1.555923 & -2.326278 \\ 1 & -3.697112 & 2.436025 & 0.147819 \\ 1 & -1.409957 & 2.843036 & 1.513604 \\ 1 & 0.652396 & 2.184433 & -0.116409 \\ 6 & -3.320298 & -1.165181 & 1.361968 \\ 6 & -4.013021 & -0.526070 & 0.293209 \\ 6 & -3.545535 & -1.087679 & -0.943566 \\ 6 & -2.587221 & -2.102061 & -0.633586 \\ 6 & -2.439267 & -2.140740 & 0.787148 \\ 1 & -3.440479 & -0.955639 & 2.415941 \\ 1 & -4.758522 & 0.250288 & 0.394475 \\ 1 & -3.890188 & -0.822476 & -1.933706 \\ 1 & -2.062079 & -2.720681 & -1.347226 \\ 1 & -1.750210 & -2.772969 & 1.329600 \\ 15 & 2.993380 & 0.596665 & 1.958682 \\ 1 & 2.882362 & -0.247588 & 3.108486 \\ 1 & 4.335335 & 1.069260 & 2.179889 \\ 1 & 2.297805 & 1.737960 & 2.478761 \\ 15 & 4.052173 & -0.210599 & -1.394442 \\ 1 & 5.315838 & 0.414088 & -1.087749 \\ 1 & 4.575148 & -1.445894 & -1.902035 \\ 1 & 3.816482 & 0.434652 & -2.653506\end{array}$




$\begin{array}{lccc}\mathbf{4 Z r}-\mathbf{N i} \\ \text { Total Energy including } & \\ \text { ZPVE }=-773.961230 \mathrm{au} & \\ \text { NIF }=0 \\ 6 & 1.574423 & -0.439580 & -1.750057 \\ 1 & 1.680883 & 0.291962 & -2.562262 \\ 1 & 1.756762 & -1.460281 & -2.112447 \\ 28 & 2.646129 & -0.155380 & -0.109239 \\ 6 & 0.775105 & -0.314848 & 0.425515 \\ 6 & 0.342575 & -0.305099 & -0.900467 \\ 40 & -1.577334 & 0.014675 & 0.031773 \\ 6 & -0.042167 & -0.191696 & 1.697326 \\ 1 & -0.032062 & -1.093068 & 2.321292 \\ 1 & 0.205366 & 0.688520 & 2.303545 \\ 6 & -1.601902 & 2.279493 & -1.260788 \\ 6 & -2.977151 & 1.916154 & -1.086189 \\ 6 & -3.283574 & 1.987143 & 0.312577 \\ 6 & -2.096105 & 2.387837 & 1.007124 \\ 6 & -1.060569 & 2.568770 & 0.034146 \\ 1 & -1.071655 & 2.335609 & -2.201452 \\ 1 & -3.671072 & 1.661859 & -1.876786 \\ 1 & -4.247657 & 1.792040 & 0.763232 \\ 1 & -2.004308 & 2.541699 & 2.073895 \\ 1 & -0.037091 & 2.852995 & 0.239816 \\ 6 & -2.788998 & -2.027102 & 1.159291 \\ 6 & -3.798697 & -1.343971 & 0.410026 \\ 6 & -3.472931 & -1.460324 & -0.983157 \\ 6 & -2.269277 & -2.231111 & -1.095492 \\ 6 & -1.846220 & -2.573605 & 0.228284 \\ 1 & -2.751162 & -2.125071 & 2.235966 \\ 1 & -4.665226 & -0.842415 & 0.820550 \\ 1 & -4.057864 & -1.072049 & -1.806873 \\ 1 & -1.773028 & -2.507721 & -2.015472 \\ 1 & -0.954110 & -3.131293 & 0.480825 \\ 15 & 3.399985 & 0.105566 & 2.086108 \\ 1 & 2.993138 & -0.939961 & 2.976662 \\ 1 & 4.760241 & 0.235133 & 2.544720 \\ 1 & 2.848706 & 1.207660 & 2.819256 \\ 15 & 4.594236 & 0.007364 & -1.348501 \\ 1 & 5.954636 & 0.202515 & -0.901203 \\ 1 & 4.844973 & -1.109750 & -2.212785 \\ 1 & 4.581878 & 1.028941 & -2.355640\end{array}$

\section{Ti-Ti}

Total Energy including ZPVE=-1043.38755 a.u.
$10.385653-2.759472$
0.000000
$6-0.066916-1.768650$
0.000000
$\begin{array}{llll}6 & 0.560648 & -0.582727 & 0.000000\end{array}$
$\begin{array}{llll}22 & -1.908108 & -0.768670 & 0.000000\end{array}$
$\begin{array}{llll}22 & 2.065313 & 0.836432 & 0.000000\end{array}$

$\begin{array}{llll}6 & 0.0 & 0.807950 & 0.000000\end{array}$

$\begin{array}{llll}6 & -1.274479 & 1.228132 & 0.000000\end{array}$

$\begin{array}{llll}1 & -1.632881 & 2.256764 & 0.000000\end{array}$

$\begin{array}{llll}1 & 4.752275 & 2.029559 & 1.204787\end{array}$

$\begin{array}{llll}6 & 3.825026 & 1.601378 & 1.558039\end{array}$

$\begin{array}{llll}6 & 3.577573 & 0.211609 & 1.794652\end{array}$

$\begin{array}{llll}1 & 2.492530 & 3.394078 & 1.773306\end{array}$

$\begin{array}{llll}1 & 4.290028 & -0.591935 & 1.660204\end{array}$

$\begin{array}{llll}6 & 2.235381 & 0.076387 & 2.281587\end{array}$

$\begin{array}{llll}1 & 1.756986 & -0.845416 & 2.574732\end{array}$

$\begin{array}{llll}6 & 1.647909 & 1.385242 & 2.320798\end{array}$

$\begin{array}{llll}1 & 0.645182 & 1.624227 & 2.639565\end{array}$

$\begin{array}{llll}6 & 2.627080 & 2.323321 & 1.857699\end{array}$

$\begin{array}{llll}1 & -3.852959 & -2.970206 & 1.203494\end{array}$

$\begin{array}{llll}6 & -3.325602 & -2.092604 & 1.548426\end{array}$

$\begin{array}{llll}6 & -3.835882 & -0.746768 & 1.556776\end{array}$

$\begin{array}{llll}1 & -1.371877 & -2.927987 & 2.252640\end{array}$

$\begin{array}{llll}1 & -4.813244 & -0.434970 & 1.217901\end{array}$

$\begin{array}{llll}6 & -2.844348 & 0.097104 & 2.134460\end{array}$

$\begin{array}{llll}1 & -2.926413 & 1.163477 & 2.288284\end{array}$

$\begin{array}{llll}6 & -1.712598 & -0.717995 & 2.461002\end{array}$

$\begin{array}{llll}1 & -0.781938 & -0.369846 & 2.884145\end{array}$

$\begin{array}{llll}6 & -2.020200 & -2.074196 & 2.116753\end{array}$

$\begin{array}{llll}1 & -0.781938 & -0.369846 & -2.884145\end{array}$

$\begin{array}{llll}6 & -1.712598 & -0.717995 & -2.461002\end{array}$

$\begin{array}{lllll}6 & -2.844348 & 0.097104 & -2.134460\end{array}$

$\begin{array}{llll}1 & -1.371877 & -2.927987 & -2.252640\end{array}$

$\begin{array}{lllll}1 & -2.926413 & 1.163477 & -2.288284\end{array}$

$\begin{array}{llll}6 & -3.835882 & -0.746768 & -1.556776\end{array}$

$\begin{array}{llll}1 & -4.813244 & -0.434970 & -1.217901\end{array}$

$\begin{array}{llll}6 & -3.325602 & -2.092604 & -1.548426\end{array}$

$1-3.852959-2.970206 \quad-1.203494$

$\begin{array}{llll}6 & -2.020200 & -2.074196 & -2.116753\end{array}$

$\begin{array}{llll}1 & 0.645182 & 1.624227 & -2.639565\end{array}$

$\begin{array}{llll}6 & 1.647909 & 1.385242 & -2.320798\end{array}$

$\begin{array}{llll}6 & 2.235381 & 0.076387 & -2.281587\end{array}$

$\begin{array}{llll}1 & 2.492530 & 3.394078 & -1.773306\end{array}$

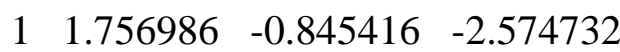

$\begin{array}{llll}6 & 3.577573 & 0.211609 & -1.794652\end{array}$

$\begin{array}{lllll}1 & 4.290028 & -0.591935 & -1.660204\end{array}$

$\begin{array}{llll}6 & 3.825026 & 1.601378 & -1.558039\end{array}$

$\begin{array}{llll}1 & 4.752275 & 2.029559 & -1.204787\end{array}$

$\begin{array}{llll}6 & 2.627080 & 2.323321 & -1.857699\end{array}$

\section{Zr-Zr}

Total Energy including ZPVE=-1020.43119 a.u.

$\begin{array}{llll}1 & 1.488244 & 2.393261 & 0.000000\end{array}$

$\begin{array}{llll}6 & 1.257056 & 1.326327 & 0.000000\end{array}$

$\begin{array}{llll}6 & 0.000000 & 0.820289 & 0.000000\end{array}$

$\begin{array}{llll}40 & 2.052313 & -0.749242 & 0.000000\end{array}$ 


$\begin{array}{rrrr}40 & -2.211295 & 0.812331 & 0.000000 \\ 6 & -0.530459 & -0.625690 & 0.000000 \\ 6 & 0.105318 & -1.822977 & 0.000000 \\ 1 & -0.406090 & -2.787418 & 0.000000 \\ 1 & -5.126576 & 1.398223 & 1.381234 \\ 6 & -4.110577 & 1.246117 & 1.721136 \\ 6 & -3.117942 & 2.267493 & 1.899052 \\ 1 & -4.022992 & -0.970337 & 2.085131 \\ 1 & -3.263799 & 3.327904 & 1.734270 \\ 6 & -1.924764 & 1.644565 & 2.405556 \\ 1 & -1.006501 & 2.147126 & 2.673994 \\ 6 & -2.181896 & 0.237828 & 2.526245 \\ 1 & -1.483599 & -0.509202 & 2.874421 \\ 6 & -3.526381 & -0.007658 & 2.097281 \\ 1 & 5.019506 & -0.369086 & 1.311816 \\ 6 & 4.052110 & -0.699922 & 1.665207 \\ 6 & 3.569050 & -2.056597 & 1.663301 \\ 1 & 3.112986 & 1.192796 & 2.417876 \\ 1 & 4.111153 & -2.923001 & 1.308781 \\ 6 & 2.271030 & -2.064795 & 2.259167 \\ 1 & 1.643914 & -2.932492 & 2.410311 \\ 6 & 1.941955 & -0.714434 & 2.611258 \\ 1 & 1.018517 & -0.384058 & 3.067348 \\ 6 & 3.050783 & 0.124667 & 2.262746 \\ 1 & 1.018517 & -0.384058 & -3.067348 \\ 6 & 1.941955 & -0.714434 & -2.611258 \\ 6 & 2.271030 & -2.064795 & -2.259167 \\ 1 & 3.112986 & 1.192796 & -2.417876 \\ 1 & 1.643914 & -2.932492 & -2.410311 \\ 6 & 3.569050 & -2.056597 & -1.663301 \\ 1 & 4.111153 & -2.923001 & -1.308781 \\ 6 & 4.052110 & -0.699922 & -1.665207 \\ 1 & 5.019506 & -0.369086 & -1.311816 \\ 6 & 3.050783 & 0.124667 & -2.262746 \\ 1 & -1.483599 & -0.509202 & -2.874421 \\ 6 & -2.181896 & 0.237828 & -2.526245 \\ 6 & -1.924764 & 1.644565 & -2.405556 \\ 1 & -4.022992 & -0.970337 & -2.085131 \\ 1 & -1.006501 & 2.147126 & -2.673994 \\ 6 & -3.117942 & 2.267493 & -1.899052 \\ 1 & -3.263799 & 3.327904 & -1.734270 \\ 6 & -4.110577 & 1.246117 & -1.721136 \\ 1 & -5.126576 & 1.398223 & -1.381234 \\ 6 & -3.526381 & -0.007658 & -2.097281\end{array}$

\section{Ti-Ni}

Total Energy including

ZPVE $=-785.439200$

$\mathrm{NIF}=0$

$\begin{array}{llll}6 & 1.201747 & -1.380903 & -1.390641\end{array}$

$\begin{array}{lccc}1 & 1.223129 & -1.118422 & -2.452745 \\ 1 & 1.552109 & -2.398916 & -1.190268 \\ 28 & 2.240824 & -0.373489 & 0.006580 \\ 6 & 0.443259 & -0.710950 & 0.759093 \\ 6 & 0.081470 & -0.911434 & -0.576206 \\ 22 & -1.593166 & 0.054260 & 0.048768 \\ 6 & -0.417920 & -0.139481 & 1.839634 \\ 1 & -0.732055 & -0.851598 & 2.607615 \\ 1 & -0.041792 & 0.778462 & 2.301814 \\ 6 & -0.934355 & 1.731059 & -1.633436 \\ 6 & -2.357998 & 1.805064 & -1.540185 \\ 6 & -2.698196 & 2.268364 & -0.229076 \\ 6 & -1.485218 & 2.483637 & 0.497044 \\ 6 & -0.397528 & 2.155826 & -0.372622 \\ 1 & -0.367225 & 1.426334 & -2.501482 \\ 1 & -3.057208 & 1.555923 & -2.326278 \\ 1 & -3.697112 & 2.436025 & 0.147819 \\ 1 & -1.409957 & 2.843036 & 1.513604 \\ 1 & 0.652396 & 2.184433 & -0.116409 \\ 6 & -3.320298 & -1.165181 & 1.361968 \\ 6 & -4.013021 & -0.526070 & 0.293209 \\ 6 & -3.545535 & -1.087679 & -0.943566 \\ 6 & -2.587221 & -2.102061 & -0.633586 \\ 6 & -2.439267 & -2.140740 & 0.787148 \\ 1 & -3.440479 & -0.955639 & 2.415941 \\ 1 & -4.758522 & 0.250288 & 0.394475 \\ 1 & -3.890188 & -0.822476 & -1.933706 \\ 1 & -2.062079 & -2.720681 & -1.347226 \\ 1 & -1.750210 & -2.772969 & 1.329600 \\ 15 & 2.993380 & 0.596665 & 1.958682 \\ 1 & 2.882362 & -0.247588 & 3.108486 \\ 1 & 4.335335 & 1.069260 & 2.179889 \\ 1 & 2.297805 & 1.737960 & 2.478761 \\ 15 & 4.052173 & -0.210599 & -1.394442 \\ 1 & 5.315838 & 0.414088 & -1.087749 \\ 1 & 4.575148 & -1.445894 & -1.902035 \\ 1 & 3.816482 & 0.434652 & -2.653506\end{array}$

\section{Zr-Ni}

Total Energy including ZPVE $=-773.961230$

$\mathrm{NIF}=0$

$\begin{array}{lccc}6 & 1.574423 & -0.439580 & -1.750057 \\ 1 & 1.680883 & 0.291962 & -2.562262 \\ 1 & 1.756762 & -1.460281 & -2.112447 \\ 28 & 2.646129 & -0.155380 & -0.109239 \\ 6 & 0.775105 & -0.314848 & 0.425515 \\ 6 & 0.342575 & -0.305099 & -0.900467 \\ 40 & -1.577334 & 0.014675 & 0.031773 \\ 6 & -0.042167 & -0.191696 & 1.697326 \\ 1 & -0.032062 & -1.093068 & 2.321292\end{array}$




$\begin{array}{lccc}1 & 0.205366 & 0.688520 & 2.303545 \\ 6 & -1.601902 & 2.279493 & -1.260788 \\ 6 & -2.977151 & 1.916154 & -1.086189 \\ 6 & -3.283574 & 1.987143 & 0.312577 \\ 6 & -2.096105 & 2.387837 & 1.007124 \\ 6 & -1.060569 & 2.568770 & 0.034146 \\ 1 & -1.071655 & 2.335609 & -2.201452 \\ 1 & -3.671072 & 1.661859 & -1.876786 \\ 1 & -4.247657 & 1.792040 & 0.763232 \\ 1 & -2.004308 & 2.541699 & 2.073895 \\ 1 & -0.037091 & 2.852995 & 0.239816 \\ 6 & -2.788998 & -2.027102 & 1.159291 \\ 6 & -3.798697 & -1.343971 & 0.410026 \\ 6 & -3.472931 & -1.460324 & -0.983157 \\ 6 & -2.269277 & -2.231111 & -1.095492 \\ 6 & -1.846220 & -2.573605 & 0.228284 \\ 1 & -2.751162 & -2.125071 & 2.235966 \\ 1 & -4.665226 & -0.842415 & 0.820550 \\ 1 & -4.057864 & -1.072049 & -1.806873 \\ 1 & -1.773028 & -2.507721 & -2.015472 \\ 1 & -0.954110 & -3.131293 & 0.480825 \\ 15 & 3.399985 & 0.105566 & 2.086108 \\ 1 & 2.993138 & -0.939961 & 2.976662 \\ 1 & 4.760241 & 0.235133 & 2.544720 \\ 1 & 2.848706 & 1.207660 & 2.819256 \\ 15 & 4.594236 & 0.007364 & -1.348501 \\ 1 & 5.954636 & 0.202515 & -0.901203 \\ 1 & 4.844973 & -1.109750 & -2.212785 \\ 1 & 4.581878 & 1.028941 & -2.355640\end{array}$

\section{Ti-Ti}

Total Energy including ZPVE = -1044.605857 au $\mathrm{NIF}=0$

$\begin{array}{lccc}6 & 0.809997 & -0.000386 & -1.812031 \\ 1 & 0.742820 & 0.895643 & -2.435182 \\ 1 & 0.744660 & -0.895902 & -2.436071 \\ 22 & 2.078737 & -0.000274 & -0.122439 \\ 6 & 0.161526 & -0.001879 & 0.6656920 \\ 6 & -0.161532 & -0.001618 & -0.665695 \\ 22 & -2.078810 & -0.000221 & 0.122405 \\ 6 & -0.810009 & -0.002238 & 1.812016 \\ 1 & -0.744766 & -0.899020 & 2.434273 \\ 1 & -0.743137 & 0.892448 & 2.437125 \\ 6 & 2.168774 & -2.169741 & 1.039750 \\ 6 & 1.898121 & -2.451341 & -0.335958 \\ 6 & 3.035852 & -2.058665 & -1.110848 \\ 6 & 4.015785 & -1.541168 & -0.210788 \\ 6 & 3.475456 & -1.594760 & 1.117028 \\ 1 & 1.509512 & -2.357781 & 1.875600 \\ 1 & 0.977308 & -2.857193 & -0.729800\end{array}$

$\begin{array}{rrrr}1 & 3.140083 & -2.148933 & -2.183097 \\ 1 & 4.999523 & -1.187056 & -0.482617 \\ 1 & 3.981778 & -1.291326 & 2.022688 \\ 6 & 4.012501 & 1.543910 & -0.213966 \\ 6 & 3.028377 & 2.062705 & -1.108724 \\ 6 & 1.893069 & 2.451382 & -0.328400 \\ 6 & 2.169368 & 2.166064 & 1.045484 \\ 6 & 3.477276 & 1.593098 & 1.116094 \\ 1 & 4.995765 & 1.192243 & -0.490612 \\ 1 & 3.128421 & 2.156166 & -2.181090 \\ 1 & 0.970161 & 2.857075 & -0.717438 \\ 1 & 1.513045 & 2.350844 & 1.884379 \\ 1 & 3.987638 & 1.287949 & 2.018900 \\ 6 & -2.158061 & 2.172138 & -1.035263 \\ 6 & -3.464528 & 1.598355 & -1.123781 \\ 6 & -4.014964 & 1.542461 & 0.199781 \\ 6 & -3.041170 & 2.056450 & 1.108497 \\ 6 & -1.897319 & 2.449991 & 0.343010 \\ 1 & -1.492396 & 2.361212 & -1.865753 \\ 1 & -3.964165 & 1.297473 & -2.034006 \\ 1 & -5.001144 & 1.188820 & 0.463159 \\ 1 & -3.153295 & 2.144273 & 2.180173 \\ 1 & -0.979168 & 2.854123 & 0.744782 \\ 6 & -3.027506 & -2.063057 & 1.110775 \\ 6 & -4.014181 & -1.541788 & 0.220313 \\ 6 & -3.484149 & -1.590548 & -1.111858 \\ 6 & -2.176703 & -2.165279 & -1.046840 \\ 6 & -1.895702 & -2.452405 & 0.325789 \\ 1 & -3.123581 & -2.157501 & 2.183404 \\ 1 & -4.995996 & -1.189381 & 0.501137 \\ 1 & -3.997613 & -1.283463 & -2.012226 \\ 1 & -1.523681 & -2.350231 & -1.888289 \\ 1 & -0.971836 & -2.859698 & 0.710859\end{array}$

\section{Zr-Zr}

Total Energy including ZPVE = -1021.653224 au $\mathrm{NIF}=0$

$\begin{array}{lccc}6 & 0.155751 & -0.055723 & -0.677011 \\ 40 & 2.229436 & -0.008373 & 0.107915 \\ 6 & 0.826902 & -0.045556 & 1.849283 \\ 1 & 0.687546 & 0.834193 & 2.486714 \\ 1 & 0.739217 & -0.950632 & 2.460453 \\ 6 & -2.340855 & 2.306334 & 1.076654 \\ 6 & -2.039841 & 2.583321 & -0.295930 \\ 6 & -3.174559 & 2.216771 & -1.090106 \\ 6 & -4.181766 & 1.712061 & -0.205760 \\ 6 & -3.664185 & 1.760047 & 1.132408 \\ 1 & -1.692619 & 2.487015 & 1.923648 \\ 1 & -1.111009 & 2.991474 & -0.671540 \\ 1 & -3.262431 & 2.321173 & -2.163149\end{array}$




$\begin{array}{rrrr}1 & -5.170127 & 1.378957 & -0.493009 \\ 1 & -4.195813 & 1.473249 & 2.030422 \\ 6 & -4.251312 & -1.636341 & -0.311138 \\ 6 & -3.212388 & -2.207112 & -1.114572 \\ 6 & -2.153142 & -2.609442 & -0.237973 \\ 6 & -2.533443 & -2.291025 & 1.106128 \\ 6 & -3.830369 & -1.683543 & 1.060509 \\ 1 & -5.199917 & -1.262348 & -0.672684 \\ 1 & -3.232194 & -2.328002 & -2.189094 \\ 1 & -1.221243 & -3.067911 & -0.541168 \\ 1 & -1.953524 & -2.487945 & 1.997872 \\ 1 & -4.408897 & -1.354279 & 1.913833 \\ 6 & 2.531309 & -2.292147 & -1.104432 \\ 6 & 3.828149 & -1.684190 & -1.062428 \\ 6 & 4.252257 & -1.635568 & 0.308208 \\ 6 & 3.215322 & -2.205737 & 1.114628 \\ 6 & 2.154182 & -2.609269 & 0.240850 \\ 1 & 1.949369 & -2.490057 & -1.994633 \\ 1 & 4.404594 & -1.355581 & -1.917421 \\ 1 & 5.201596 & -1.260997 & 0.667223 \\ 1 & 3.237610 & -2.325449 & 2.189239 \\ 1 & 1.223108 & -3.067676 & 0.546670 \\ 6 & 3.179629 & 2.214612 & 1.089900 \\ 6 & 4.183003 & 1.711186 & 0.200484 \\ 6 & 3.659816 & 1.761422 & -1.135425 \\ 6 & 2.336956 & 2.308177 & -1.073217 \\ 6 & 2.041682 & 2.582828 & 0.301045 \\ 1 & 3.272002 & 2.317112 & 2.162758 \\ 1 & 5.172478 & 1.377361 & 0.483003 \\ 1 & 4.187543 & 1.475976 & -2.036177 \\ 1 & 1.685231 & 2.490359 & -1.917195 \\ 1 & 1.114530 & 2.990533 & 0.681259\end{array}$

6

6

6

6

6

6

6

6

6

6

6

6

6

6

6

6

\section{1}

\section{1}

$$
1
$$

$$
1
$$$$
1
$$$$
1
$$$$
1
$$$$
1
$$$$
1
$$

\section{$\mathrm{Cp}_{2} \operatorname{Zr}\left[\left(\mu-\eta^{4} \mathrm{H}_{2} \mathrm{C}_{4} \mathrm{H}_{2}\right)\right] \mathrm{ZrCp}_{2}\left(\mathrm{PMe}_{3}\right)$}

Total Energy including $\mathrm{ZPVE}=-1147.766627 \mathrm{au}$ $\mathrm{NIF}=0$

$\begin{array}{lrrr}40 & -2.643450 & -0.023390 & 0.034270 \\ 40 & 1.912414 & -0.037632 & -0.607401 \\ 15 & 2.721371 & -0.018332 & 2.074346 \\ 6 & -1.020153 & -0.390567 & 1.623997 \\ 6 & -0.102131 & -0.272764 & 0.445307 \\ 6 & -0.282648 & -0.267159 & -0.903850 \\ 6 & -1.407622 & -0.339032 & -1.878490 \\ 6 & -2.048300 & 2.513942 & 0.001184 \\ 6 & -2.743659 & 2.290627 & 1.233726 \\ 6 & -4.092554 & 1.934221 & 0.916689 \\ 6 & -4.231381 & 1.946775 & -0.512919 \\ 6 & -2.968695 & 2.311584 & -1.077997\end{array}$

\begin{tabular}{ccc}
-3.026428 & -2.606063 & 0.034618 \\
-3.573853 & -2.141030 & 1.276013 \\
-4.698548 & -1.314060 & 0.979261 \\
-4.866394 & -1.288305 & -0.450291 \\
-3.843541 & -2.098027 & -1.029198 \\
1.736850 & 2.549789 & -0.401146 \\
1.306483 & 2.221462 & -1.727500 \\
2.443440 & 1.733497 & -2.449881 \\
3.583507 & 1.788669 & -1.581964 \\
3.147982 & 2.283219 & -0.316708 \\
1.706724 & -2.293372 & -1.884570 \\
2.813483 & -1.608967 & -2.484774 \\
3.883058 & -1.564141 & -1.530627 \\
3.430563 & -2.206058 & -0.336808 \\
2.082616 & -2.653044 & -0.550310 \\
2.069064 & 1.415077 & 3.121538 \\
4.570017 & 0.060200 & 2.500484 \\
2.181084 & -1.504546 & 3.107707 \\
-0.802395 & 0.304338 & 2.442449 \\
-1.074452 & -1.409018 & 2.028747 \\
-1.326076 & 0.386342 & -2.695699 \\
-1.543594 & -1.338547 & -2.309450 \\
-1.012032 & 2.797086 & -0.097137 \\
-2.329492 & 2.389117 & 2.227241 \\
-4.879550 & 1.728527 & 1.629633 \\
-5.140071 & 1.750291 & -1.065723 \\
-2.755172 & 2.426851 & -2.131157 \\
-2.165036 & -3.252088 & -0.078713 \\
-3.205691 & -2.375866 & 2.264606 \\
-5.340688 & -0.831623 & 1.703844 \\
-5.656270 & -0.782391 & -0.988864 \\
-3.714232 & -2.295504 & -2.083751 \\
1.126018 & 2.970826 & 0.385261 \\
0.309441 & 2.337215 & -2.126984 \\
2.448487 & 1.424543 & -3.487076 \\
4.595785 & 1.508376 & -1.841785 \\
3.785438 & 2.488415 & 0.533117 \\
0.762999 & -2.510951 & -2.36317 \\
2.850596 & -1.233947 & -3.499203 \\
4.866258 & -1.143067 & -1.694482 \\
4.026765 & -2.379781 & 0.549379 \\
1.466307 & -3.185727 & 0.161449 \\
0.976161 & 1.401567 & 3.100815 \\
2.415364 & 1.325756 & 4.158146 \\
2.416901 & 2.366397 & 2.707262 \\
4.720941 & 0.057308 & 3.586708 \\
5.087716 & -0.800574 & 2.064796 \\
1.092897 & -1.588524 & 3.049852 \\
\hline .488398 & -1.384038 & 4.1533970
\end{tabular}




\section{3-5-Ti-Ni-TS}

Total Energy including

ZPVE $=-785.414841 \mathrm{au}$

$\mathrm{NIF}=0$$$
1
$$

\section{6}

6

6

$$
-0.70958
$$

$-0.396839$

0.485473

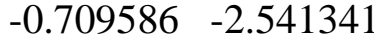

$-0.595674-2.846606$

$-1.098718-3.293652$

0.136150

1.175819

$-0.192201-1.339860$

$-0.557502$

$-0.470784$

$-0.299435$

0.440382

1.173275

0.002097

0.075187

1

28

22

6

6

6

6

6

1.696083

0.997529

2.669618

\section{3-5-Zr-Ni-TS}

Total Energy including ZPVE $=-773.946324$ $\mathrm{NIF}=1$

$\begin{array}{rrrr}6 & -0.227457 & -1.064343 & 2.462593 \\ 1 & 0.348418 & -0.593277 & 3.257463 \\ 1 & -0.741202 & -1.993929 & 2.725312 \\ 6 & -0.287520 & -0.531343 & 1.206882\end{array}$$$
1
$$

28

40

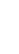

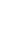

6

6

\section{6}

\section{6}

\section{1}

15

\section{1}

\section{3-5-Ti-Ti-TS}

T otal Energy including ZPVE $=-1044.559664$

\section{$\mathrm{NIF}=1$}

$\begin{array}{lrcc}6 & -0.257456 & -0.135079 & 0.951263 \\ 6 & -0.018509 & -0.158851 & -0.376451 \\ 6 & 0.823737 & -0.224717 & 1.9376600 \\ 6 & -0.043247 & -0.150430 & -1.782902 \\ 22 & 2.030815 & -0.010579 & 0.049893 \\ 22 & -2.094861 & -0.017208 & -0.084359 \\ 1 & 0.850078 & 0.582201 & 2.673264 \\ 1 & 0.919904 & -1.192413 & 2.432684 \\ 1 & -0.252768 & 0.757918 & -2.338925 \\ 1 & 0.182834 & -1.043659 & -2.356599 \\ 6 & 1.970941 & -2.401394 & -0.465916 \\ 1 & 1.023714 & -2.882071 & -0.663409\end{array}$




$\begin{array}{llll}6 & 2.816927 & -1.765522 & -1.439812 \\ 1 & 2.627713 & -1.693223 & -2.501507 \\ 6 & 3.975438 & -1.283319 & -0.762130 \\ 1 & 4.806098 & -0.766065 & -1.220621 \\ 6 & 2.615675 & -2.308022 & 0.804397 \\ 1 & 2.255095 & -2.723638 & 1.732854 \\ 6 & 3.841589 & -1.593642 & 0.631139 \\ 1 & 4.556444 & -1.364931 & 1.409744 \\ 6 & 1.715510 & 2.393341 & 0.549532 \\ 1 & 0.777650 & 2.726994 & 0.967504 \\ 6 & 2.853916 & 1.938627 & 1.298065 \\ 1 & 2.936083 & 1.904363 & 2.374768 \\ 6 & 3.875327 & 1.585080 & 0.366434 \\ 1 & 4.862370 & 1.222905 & 0.616238 \\ 6 & 2.032859 & 2.303513 & -0.837554 \\ 1 & 1.388740 & 2.569815 & -1.663111 \\ 6 & 3.358776 & 1.778587 & -0.957276 \\ 1 & 3.890903 & 1.613040 & -1.883633 \\ 6 & -2.485085 & -2.388724 & -0.819054 \\ 1 & -1.995294 & -2.783919 & -1.699167 \\ 6 & -2.011406 & -2.474604 & 0.520494 \\ 1 & -1.082038 & -2.927289 & 0.837602 \\ 6 & -2.940523 & -1.795064 & 1.376761 \\ 1 & -2.867269 & -1.701892 & 2.451527 \\ 6 & -3.729918 & -1.674457 & -0.808633 \\ 1 & -4.357205 & -1.478438 & -1.66744 \\ 6 & -4.020447 & -1.333686 & 0.552051 \\ 1 & -4.907057 & -0.824901 & 0.901982 \\ 6 & -1.781164 & 2.330444 & -0.511162 \\ 1 & -0.823680 & 2.680105 & -0.866152 \\ 6 & -2.882215 & 1.916369 & -1.338758 \\ 1 & -2.906030 & 1.907476 & -2.419854 \\ 6 & -3.965112 & 1.578754 & -0.474382 \\ 1 & -4.935259 & 1.217926 & -0.787709 \\ 6 & -2.185321 & 2.233667 & 0.860951 \\ 1 & -1.594812 & 2.489663 & 1.729242 \\ 6 & -3.531357 & 1.755071 & 0.880498 \\ 1 & -4.120790 & 1.565703 & 1.767681\end{array}$

\section{3-5-Zr-Zr-TS}

Total Energy including ZPVE $=-1021.609580$ $\mathrm{NIF}=1$

$\begin{array}{lccc}6 & 0.281846 & -0.347599 & 0.973386 \\ 6 & 0.031319 & -0.357109 & -0.373005 \\ 6 & -0.804548 & -0.512051 & 1.960264 \\ 6 & -0.229258 & -0.366717 & -1.773764 \\ 40 & -2.128002 & -0.031117 & 0.014597 \\ 40 & 2.213142 & -0.047646 & -0.117599 \\ 1 & -0.992867 & -1.549811 & 2.253677 \\ 1 & -0.736527 & 0.125921 & 2.845582\end{array}$

$\begin{array}{llll}1 & 0.136281 & 0.445188 & -2.407727 \\ 1 & -0.467767 & -1.292412 & -2.294106 \\ 6 & -1.646105 & 2.459579 & 0.587227 \\ 1 & -0.662191 & 2.723295 & 0.944001 \\ 6 & -2.077958 & 2.447790 & -0.776533 \\ 1 & -1.486143 & 2.712374 & -1.642401 \\ 6 & -3.452134 & 2.039941 & -0.801159 \\ 1 & -4.072049 & 1.959135 & -1.684272 \\ 6 & -2.754480 & 2.066978 & 1.410624 \\ 1 & -2.751067 & 2.001488 & 2.489713 \\ 6 & -3.873962 & 1.825986 & 0.553235 \\ 1 & -4.867847 & 1.540771 & 0.870445 \\ 6 & -2.423069 & -2.582943 & -0.358280 \\ 1 & -1.540804 & -3.205333 & -0.427417 \\ 6 & -3.157359 & -2.293837 & 0.838434 \\ 1 & -2.933892 & -2.662455 & 1.829068 \\ 6 & -4.257886 & -1.452439 & 0.490789 \\ 1 & -5.008784 & -1.078188 & 1.173872 \\ 6 & -3.092883 & -1.940056 & -1.455100 \\ 1 & -2.811982 & -1.995143 & -2.497652 \\ 6 & -4.219715 & -1.234296 & -0.930004 \\ 1 & -4.937459 & -0.665730 & -1.505997 \\ 6 & 1.760698 & 2.423232 & -0.574742 \\ 1 & 0.790235 & 2.716802 & -0.946545 \\ 6 & 2.156061 & 2.355379 & 0.803701 \\ 1 & 1.543457 & 2.582181 & 1.664762 \\ 6 & 3.536245 & 1.970144 & 0.842196 \\ 1 & 4.133381 & 1.846443 & 1.736424 \\ 6 & 2.903302 & 2.091291 & -1.385120 \\ 1 & 2.943170 & 2.096586 & -2.466969 \\ 6 & 4.000160 & 1.825663 & -0.506773 \\ 1 & 5.004851 & 1.561378 & -0.808251 \\ 6 & 2.411718 & -2.612453 & -0.07901 \\ 1 & 1.536208 & -3.188600 & -0.346674 \\ 6 & 3.434308 & -2.163186 & -0.982833 \\ 1 & 3.476294 & -2.356876 & -2.047373 \\ 6 & 4.418261 & -1.459962 & -0.218293 \\ 1 & 5.327024 & -1.019126 & -0.605966 \\ 6 & 2.763142 & -2.181576 & 1.243664 \\ 1 & 2.203921 & -2.377107 & 2.147739 \\ 6 & 3.997307 & -1.459568 & 1.15471 \\ 1 & 4.538867 & -1.023397 & 1.984484\end{array}$

\section{3-Zr-Zr-TS}

Total Energy including ZPVE = -1021.635647 $\mathrm{NIF}=1$

$\begin{array}{lccc}6 & 0.262724 & -0.000199 & -0.683858 \\ 6 & 0.262781 & -0.000198 & 0.683382 \\ 6 & -0.765207 & -0.000489 & -1.773759 \\ 6 & -0.764935 & -0.000515 & 1.773457\end{array}$




$\begin{array}{lrrr}40 & -2.227081 & -0.000024 & -0.000035 \\ 40 & 2.361507 & -0.000084 & -0.000289 \\ 1 & -0.699512 & 0.887267 & -2.414404 \\ 1 & -0.699594 & -0.888727 & -2.413752 \\ 1 & -0.699073 & -0.888615 & 2.413617 \\ 1 & -0.699262 & 0.887374 & 2.413924 \\ 6 & -2.078594 & -2.598679 & 0.000154 \\ 1 & -1.100472 & -3.061604 & 0.000339 \\ 6 & -2.846602 & -2.254511 & 1.161364 \\ 1 & -2.557292 & -2.416490 & 2.189688 \\ 6 & -4.071174 & -1.665929 & 0.719335 \\ 1 & -4.875725 & -1.322100 & 1.355447 \\ 6 & -2.846301 & -2.254712 & -1.161308 \\ 1 & -2.556777 & -2.416906 & -2.189536 \\ 6 & -4.070981 & -1.666036 & -0.719698 \\ 1 & -4.875371 & -1.322321 & -1.356074 \\ 6 & -2.077104 & 2.598685 & 0.000206 \\ 1 & -1.098741 & 3.061097 & 0.000249 \\ 6 & -2.845139 & 2.255098 & -1.161150 \\ 1 & -2.555654 & 2.417150 & -2.189413 \\ 6 & -4.070074 & 1.667067 & -0.719364 \\ 1 & -4.874725 & 1.323751 & -1.355627 \\ 6 & -2.845107 & 2.254880 & 1.161517 \\ 1 & -2.555603 & 2.416764 & 2.189801 \\ 6 & -4.070055 & 1.666935 & 0.719661 \\ 1 & -4.874691 & 1.323521 & 1.355888 \\ 6 & 2.306725 & -2.479234 & -0.715721 \\ 1 & 1.499675 & -2.813552 & -1.352862 \\ 6 & 3.581842 & -1.987263 & -1.158512 \\ 1 & 3.904759 & -1.892337 & -2.188423 \\ 6 & 4.376032 & -1.701194 & -0.001171 \\ 1 & 5.394496 & -1.336009 & -0.002488 \\ 6 & 2.308054 & -2.477951 & 0.718717 \\ 1 & 1.502295 & -2.811391 & 1.357951 \\ 6 & 3.584099 & -1.985240 & 1.158200 \\ 1 & 3.909067 & -1.888649 & 2.187307 \\ 6 & 2.307882 & 2.477964 & 0.718255 \\ 1 & 1.501982 & 2.811487 & 1.357269 \\ 6 & 3.583856 & 1.985359 & 1.158059 \\ 1 & 3.908624 & 1.888985 & 2.187249 \\ 6 & 4.375996 & 1.701089 & -0.001113 \\ 1 & 5.394463 & 1.335910 & -0.002153 \\ 6 & 2.306784 & 2.478932 & -0.716175 \\ 1 & 1.499862 & 2.813108 & -1.353553 \\ 6 & 3.582011 & 1.986939 & -1.158644 \\ 1 & 3.905135 & 1.891820 & -2.188473\end{array}$

\section{3-Ti-Ti-TS}

Total Energy including ZPVE = -1044.591262

\begin{tabular}{|c|c|c|c|}
\hline \multicolumn{4}{|c|}{$\mathrm{NIF}=1$} \\
\hline 6 & 0.000000 & 0.671670 & 0.280344 \\
\hline 6 & 0.000000 & -0.671670 & 0.280344 \\
\hline 6 & 0.005666 & 1.741279 & -0.730511 \\
\hline 6 & -0.005666 & -1.741279 & -0.730510 \\
\hline 22 & 0.0 .00000 & 0.000000 & -2.074417 \\
\hline 22 & 0.000000 & 0.00000 & 2.236546 \\
\hline 1 & -0.900251 & 2.354794 & -0.728951 \\
\hline 1 & 0.878058 & 2.398189 & -0.68530 \\
\hline 1 & 0.900251 & -2.354794 & -0.728951 \\
\hline 1 & -0.878058 & -2.398189 & -0.685305 \\
\hline 6 & 2.445516 & -0.350174 & -1.922609 \\
\hline 1 & 2.886326 & -0.623893 & -0.974353 \\
\hline 6 & 1.944290 & -1.262307 & -2.91232 \\
\hline 1 & 1.949535 & -2.340700 & -2.849332 \\
\hline 6 & 1.451958 & -0.491891 & -4.003146 \\
\hline 1 & 1.018888 & -0.886123 & -4.911126 \\
\hline 6 & 2.277490 & 0.977869 & -2.418583 \\
\hline 1 & 2.585081 & 1.886682 & -1.924103 \\
\hline 6 & 1.631439 & 0.897867 & -3.688682 \\
\hline 1 & 1.369528 & 1.736639 & -4.31841 \\
\hline 6 & -2.445516 & 0.350174 & -1.922609 \\
\hline 1 & -2.886326 & 0.623893 & -0.974353 \\
\hline 6 & -1.944290 & 1.262307 & -2.91232 \\
\hline 1 & -1.949535 & 2.340700 & -2.849332 \\
\hline 6 & -1.451958 & 0.491891 & -4.003146 \\
\hline 1 & -1.018888 & 0.886123 & -4.911126 \\
\hline 6 & -2.277490 & -0.977869 & -2.418583 \\
\hline 1 & -2.585081 & -1.886682 & -1.924103 \\
\hline 6 & -1.631439 & -0.897867 & -3.688682 \\
\hline 1 & -1.369528 & -1.736639 & -4.31841 \\
\hline 6 & 2.435269 & 0.482494 & 2.014391 \\
\hline 1 & 2.813810 & 0.864085 & 1.077203 \\
\hline 6 & 1.968185 & 1.275992 & 3.106656 \\
\hline 1 & 1.962731 & 2.358143 & 3.149199 \\
\hline 6 & 1.527152 & 0.390477 & 4.142123 \\
\hline 1 & 1.126942 & 0.687455 & 5.101475 \\
\hline 6 & 2.248544 & -0.895720 & 2.347992 \\
\hline 1 & 2.519111 & -1.744693 & 1.735723 \\
\hline 6 & 1.680551 & -0.951745 & 3.670567 \\
\hline 1 & 1.454393 & -1.851721 & 4.227242 \\
\hline 6 & -2.435269 & -0.482494 & 2.014391 \\
\hline 1 & -2.813810 & -0.864085 & 1.077203 \\
\hline 6 & -1.968185 & -1.275992 & 3.106656 \\
\hline 1 & -1.962731 & -2.358143 & 3.149199 \\
\hline 6 & -1.527152 & -0.390477 & 4.142123 \\
\hline 1 & -1.126942 & -0.687455 & 5.101475 \\
\hline 6 & -2.248544 & 0.895720 & 2.347992 \\
\hline 1 & -2.519111 & 1.744693 & 1.735723 \\
\hline 6 & -1.680551 & 0.951745 & 3.670567 \\
\hline 1 & -1.454393 & 1.851721 & 4.227242 \\
\hline
\end{tabular}




\begin{tabular}{lccc}
\multicolumn{4}{l}{ 3-Zr-Ni-TS } \\
\multicolumn{4}{l}{ Total Energy including } \\
ZPVE $=-773.998650$ & \\
NIF = 1 & & \\
6 & 0.025572 & 0.001426 & -1.823186 \\
1 & -0.039852 & -0.893918 & -2.449093 \\
1 & -0.040316 & 0.898582 & -2.446485 \\
6 & -0.887765 & -0.000372 & -0.665413 \\
6 & -0.887763 & -0.002311 & 0.665275 \\
6 & 0.025511 & -0.003964 & 1.823094 \\
1 & -0.041170 & 0.890698 & 2.449842 \\
1 & -0.039164 & -0.901805 & 2.445537 \\
6 & 1.342761 & -2.589199 & 0.001458 \\
1 & 0.359478 & -3.040670 & 0.004979 \\
6 & 2.116743 & -2.246631 & 1.159633 \\
1 & 1.830774 & -2.407385 & 2.189051 \\
6 & 3.344398 & -1.666445 & 0.712972 \\
1 & 4.151684 & -1.323929 & 1.346443 \\
6 & 3.339471 & -1.667027 & -0.724468 \\
1 & 4.142289 & -1.325059 & -1.363874 \\
6 & 2.108926 & -2.247950 & -1.162161 \\
1 & 1.816095 & -2.409957 & -2.189429 \\
6 & 2.113270 & 2.247759 & -1.159605 \\
1 & 1.826946 & 2.408185 & -2.188978 \\
6 & 3.341799 & 1.669279 & -0.713104 \\
1 & 4.149499 & 1.327947 & -1.346683 \\
6 & 3.337023 & 1.669762 & 0.724332 \\
1 & 4.140392 & 1.328893 & 1.363630 \\
6 & 2.105697 & 2.248918 & 1.162198 \\
1 & 1.812728 & 2.410461 & 2.189504 \\
6 & 1.338972 & 2.589224 & -0.001325 \\
1 & 0.355073 & 3.039347 & -0.004716 \\
1 & -3.771637 & -1.078279 & 2.811689 \\
1 & -3.769275 & 1.068892 & 2.815545 \\
1 & -5.406522 & -0.001331 & 1.930292 \\
1 & -5.406928 & -0.008959 & -1.929611 \\
1 & -3.780352 & 1.084827 & -2.805497 \\
1 & -3.762156 & -1.062204 & -2.821387 \\
15 & -3.963596 & -0.002788 & 1.873576 \\
15 & -3.964032 & 0.002679 & -1.873357 \\
40 & 1.485105 & 0.000052 & -0.000021 \\
28 & -2.700181 & -0.000700 & -0.000050
\end{tabular}




\section{Complete Authors list for reference No. 11}

Gaussian 03, Revision C.02, Frisch, M. J.; Trucks, G. W.; Schlegel, H. B.; Scuseria, G. E.; Robb, M. A.; Cheeseman, J. R.; Montgomery, Jr., J. A.; Vreven, T.; Kudin, K. N.; Burant, J. C.; Millam, J. M.; Iyengar, S. S.; Tomasi, J.; Barone, V.; Mennucci, B.; Cossi, M.; Scalmani, G.; Rega, N.; Petersson, G. A.; Nakatsuji, H.; Hada, M.; Ehara, M.; Toyota, K.; Fukuda, R.; Hasegawa, J.; Ishida, M.; Nakajima, T.; Honda, Y.; Kitao, O.; Nakai, H.; Klene, M.; Li, X.; Knox, J. E.; Hratchian, H. P.; Cross, J. B.; Bakken, V.; Adamo, C.; Jaramillo, J.; Gomperts, R.; Stratmann, R. E.; Yazyev, O.; Austin, A. J.; Cammi, R.; Pomelli, C.; Ochterski, J. W.; Ayala, P. Y.; Morokuma, K.; Voth, G. A.; Salvador, P.; Dannenberg, J. J.; Zakrzewski, V. G.; Dapprich, S.; Daniels, A. D.; Strain, M. C.; Farkas, O.; Malick, D. K.; Rabuck, A. D.; Raghavachari, K.; Foresman, J. B.; Ortiz, J. V.; Cui, Q.; Baboul, A. G.; Clifford, S.; Cioslowski, J.; Stefanov, B. B.; Liu, G.; Liashenko, A.; Piskorz, P.; Komaromi, I.; Martin, R. L.; Fox, D. J.; Keith, T.; Al-Laham, M. A.; Peng, C. Y.; Nanayakkara, A.; Challacombe, M.; Gill, P. M. W.; Johnson, B.; Chen, W.; Wong, M. W.; Gonzalez, C.; Pople, J. A. Gaussian, Inc., Wallingford CT, 2004. 